\title{
Editorial
}

\section{Damage and fracture of biological and biomedical materials}

In the last decade, the topic of damage and fracture of biological and biomedical materials not only became one of the central research areas in the healthcare engineering, but also drew attention of specialists in mechanics of materials and fracture. One of the motivations behind these developments is a continuing increase in the use of medical devices made of various materials that are exposed to challenging loading and environmental conditions. Many of them should have significant levels of durability to avoid recurring surgical interventions (typical examples being implants for hip and knee replacements or dental implants). A lack of understanding of their responses to specific conditions and interaction with biological environment can result in malfunctioning and failures or traumas to surrounding tissues. The typical application problems are additionally complicated by insufficient knowledge of mechanical behaviour of biomaterials at various length and time scales and under different loading conditions including their fracture and fatigue.

These types of application presuppose the understanding of properties and performance of two classes of materials - natural (biomaterials) and engineering (biomedical materials), as well as their interaction at interfaces between, on the one hand, life tissues (or organs) and, on the other hand, implants and prostheses. Among engineering materials, used in such applications, are familiar metals and alloys, ceramics, polymers and composites. Their properties and performance seem to be well studied; still, biomedical applications are characterised by rather specific usability envelopes as well as, in most cases, additional constraints such as nontoxicity (biocompatibility) and/or resistance to harsh physiological environments. In some cases, a requirement, opposite to structural integrity, is needed, e.g. controlled degradation for scaffolds and stents.

Our understanding of mechanics of biological tissues, their properties and performance remains limited. This is a result of multiple factors, most important being their hierarchical and heterogeneous nature, non-trivial loading and environmental conditions, to which they are exposed, as well as multi-disciplinary nature of the systems involved. Two main types of biological materials and tissues - hard (bones) and soft (muscles, etc.), demonstrating significant differences in their mechanical properties (such as stiffness and deformability), have also two important common features, affecting their mechanical analysis. The first is their highly hierarchical microstructure that can also contain constituents with significantly different properties (e.g. soft collagen and brittle hydroxyapatite in bones). The second is a considerable variability of the local macroscopic mechanical properties not only between different species and their individual representatives, but also within the same tissue or organ. These can also be affected by the age, nutrition, disease, medical treatments and other factors. Obviously, for long-term (non-instantaneous) loading regimes, typical for many applications, the ability of biotissues to grow (remodel) should also be considered, making already complex modelling schemes even more cumbersome and experimental identification of their parameters more challenging.

Obviously, a single special issue cannot cover all these topics and provide answers to all the challenges. Still, it is an important step forward in our understanding of such non-trivial mechanical systems. This Special Issue is based on selection of papers presented at Symposium "Damage and Fracture of Biological and Biomedical Materials" (Symposium Chair - Prof. V. Silberschmidt), organised within the framework of the 22nd European Conference on Fracture on 26-31 August 2018, Belgrade, Serbia, and invited contributions by leading teams working in this area. The issue covers a good cross-section of materials, topics and challenges discussed above and presents results of theoretical, experimental and numerical studies.

Its contributions dedicated to biological materials cover both soft tissues - monolayers of endothelial cells - and hard ones: cortical and trabecular bones. Not all the contributions are motivated by medical applications. Two papers deal with other matters: crack propagation in an insect wing and indentation-induced damage evolution in millet seeds. In the domain of mechanics of biomedical materials, two papers are focused on 3d-printed biopolymers, reflecting a strong current drive of personalised medical solutions, where additive manufacturing technologies (including filament deposition) are destined to play a crucial role. So, elucidation of the effects of the architecture and microstructural features of 3d-printed designs on their mechanical properties and performance in these papers is rather timely.

A bulk of manuscripts focused on biomedical materials is inevitably motivated by, and deals (directly or indirectly) with, medical 
applications involving combinations of (mostly stiff) medical devices and surrounding tissues. In this issue, two papers present research into hip implants - their fatigue and fracture behaviours, while one considers the effect of cement on osteoporotic femoral augmentation. A similar contrast of mechanical properties of implanted materials and surrounding tissues is characteristic for the problem discussed in a paper, dealing with metallic dental bridges, manufactured with additive technologies. A much larger mismatch in properties is typical for another class of mechanical devices - stents: they are usually made of metals and alloys (nitinol in the analysed case of endovascular stents) surrounded by soft tissues, with stiffnesses lower by orders of magnitude.

The methods of analysis and simulations, presented in this collection, are not limited to their specific applications, mostly related to the field of biomedical engineering. Oppositely, they can be of interest to all the specialists in the area of engineering fracture mechanics.

Vadim V. Silberschmidt ${ }^{\mathrm{a}}$, Jose Manuel Garcia Aznar ${ }^{\mathrm{b}}$

${ }^{a}$ Wolfson School of Mechanical, Electrical and Manufacturing Engineering, Loughborough University, UK ${ }^{\mathrm{b}}$ Aragón Institute of Engineering Research (I3A), Mechanical Engineering Department, University of Zaragoza, Spain 